\title{
On D-decomposition of periodically sampled systems
}

\begin{abstract}
The problem of the stability of non-uniformly sampled systems is considered. For this purpose, the D-decomposition method for determining the stability region in parameter space is investigated. Moreover, basic information about non-uniform sampling are presented, with an emphasis on periodic sampling. Based on the obtained simulation results, some comparisons of systems with different sampling patterns are considered.
\end{abstract}

Keywords: periodic sampling, hybrid systems, D-decomposition stability

\section{Introduction}

Generally, the sampling process is described as follows:

$$
x_{s}(t)=x(t) \sum_{k=0}^{\infty} \delta\left(t-t_{k}\right)
$$

where $\delta$ denotes the Dirac impulse, $t_{k}$ are sampling instants, which can be described in the uniform sampling case as $t_{k}=k T$, where $T$ denotes the sampling period, $k \in \mathbb{N}$, and $t_{k}<t_{k+1}$; see [1]. In non-uniform sampling, the period may differ for two consecutive samples; thus, in non-uniform sampling, $t_{k} \neq k T$.

Over the last decades, many non-uniform sampling schemes have been investigated. The most-common non-uniform sampling schemes are as follows: jittered random sampling (jrs), additive random sampling (ars), recurrent sampling, periodic sampling, and multi-rate sampling; see, for example, [1-3].

The use of the practical application of non-uniform sampling has risen over the last years due to its advantages, such as decreasing data size with simultaneously ensuring sufficient accuracy; see, for example, [4]. Currently, non-uniform sampling is applied in such areas as networked control systems, medicine, and automotive applications; see, for example, [5].

Nevertheless, there are still some open problems in the non-uniform sampling theory; for example, ensuring the stability in non-uniformly sampled systems. There exist less number

\footnotetext{
* Bialystok University of Technology, Department of Automatics and Robotics, Wiejska 45C, 15-351 Białystok, Poland, e-mail: j.janczak@doktoranci.pb.edu.pl
} 
of stability results for nonuniform sampling than for uniform sampling. This work investigates the problem of the stability of a non-uniformly sampled system with the use of the D-decomposition method; see, for example, $[6,7]$. The idea of D-decomposition is based on determining the regions on a parameter plane obtained from a characteristic equation with simple parametrization by $j \omega$. In each region, there is a known number of characteristic equation roots with positive and negative real parts. This technique is based on the decomposition of the parameter space into domains with boundaries defined by $P(j \omega, \lambda)=0, \omega \in(-\infty, \infty)$ for continuous-time systems and $P\left(e^{j \omega}, \lambda\right)=0, \omega \in[0,2 \pi)$ for discrete ones; $\lambda \in \mathbb{R}^{m}$ is a parameter, and $P(s, \lambda)$ denotes an $n$ th-degree polynomial. In this paper, D-decomposition for state-space form of the system with periodic sampling of the $L$ th order is introduced; therefore, a sampled system is obtained.

The paper is organized as follows. In Section 2, the periodic sampling scheme of the 2nd and $L$ th orders is described. The basic notation and facts about D-decomposition are presented. In Section 3, simulation results based on the example of a DC motor are investigated. In Section 4, conclusions and suggestions for future works are mentioned.

\section{Periodic sampling scheme}

In this section, the periodic sampling scheme is discussed. Further basics about D-decomposition are introduced with reference to non-uniformly sampled systems. An exemplary sampling scheme that was used in the next part of this work is a periodic sampling of the $L$ th order.

Periodic sampling of the 2 nd order is a particular case of periodic sampling of the $L$ th order; both schemes can be described as follows.

1) Periodic sampling of 2 nd order:

The simplest case of non-uniform sampling occurs when two uniform samples with sampling period $T$ are interleaved by time offset $0<d_{1}<T$. This mode of sampling is called periodic sampling. The number of interleaved samples define the order of the sampling: in the 2 nd order of periodic sampling, two different lengths of sampling periods occur. The two sets of samples can be described as $x(k T), k \in \mathbb{N}$ and $x\left(k T+d_{1}\right), k \in \mathbb{N}, d_{1}<T$; see, for example, [2], which is clarified in Figure 1.

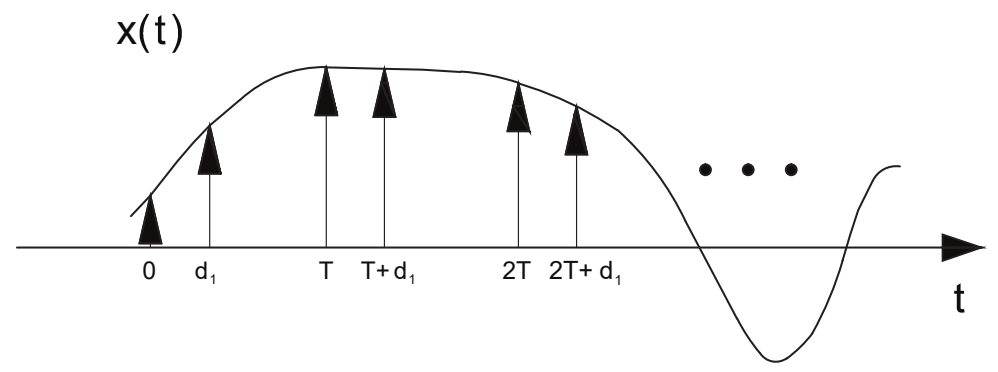

Fig. 1. Periodic sampling of second order 
2) Periodic sampling of $L$ th order:

In periodic sampling of the $L$ th order (where $L>1$ ), $L$ different sampling periods are defined; i.e., as the following set of time instance samplings $x(k T), k \in \mathbb{N}, x\left(k T+d_{1}\right), k \in \mathbb{N}$, $\ldots, x\left(k T+d_{L-1}\right), k \in \mathbb{N}$, which is presented in Figure 2.

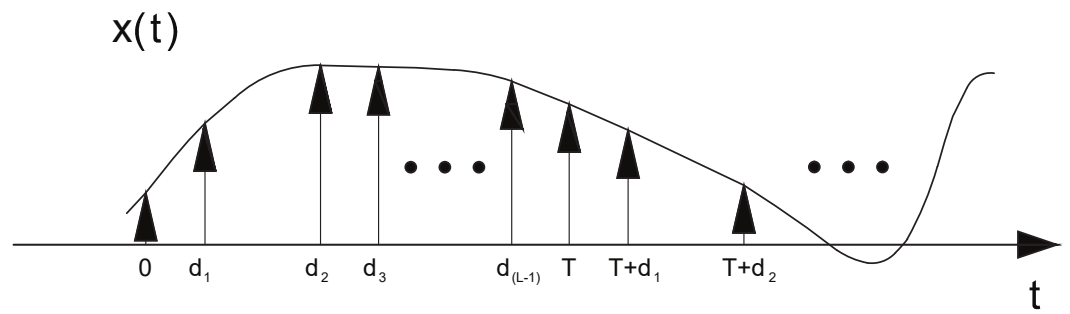

Fig. 2. Periodic sampling of $L$ th order

\section{D-decomposition theory with periodic sampling of $L$ th order}

Consider a hybrid system; i.e., mixed continuous and discrete time subsystems. The continuous-time part is defined as follows:

$$
\begin{aligned}
& \dot{x}(t)=A_{c} x(t)+B_{c} u(t) \\
& y(t)=C_{c} x(t)
\end{aligned}
$$

where $x \in \mathbb{R}^{n}$ denotes a state vector, $u \in \mathbb{R}^{m}$ a control vector, and $y \in \mathbb{R}^{r}$ an output vector, and the system matrices have the following dimensions: $A_{c} \in \mathbb{R}^{n \times n}, B_{c} \in \mathbb{R}^{n \times m}, C_{c} \in \mathbb{R}^{r \times n}$.

By non-uniformly sampling the continuous-time dynamics of (2), the following discretetime subsystem at time instants $t=t_{i}, i=1, \ldots, k$ is obtained:

$$
\begin{aligned}
& x\left(t_{i+1}\right)=A_{d_{i}} x\left(t_{i}\right)+B_{d_{i}} u\left(t_{i}\right) \\
& y\left(t_{i}\right)=C_{d_{i}} x\left(t_{i}\right)
\end{aligned}
$$

where $A_{d_{i}}, B_{d_{i}}, C_{d_{i}}$ are discrete-time system matrices of appropriate dimensions.

The discrete-time system matrix $A_{d_{i}}$ from (3) can be described as follows (see [8]):

$$
A_{d_{i}}:=\frac{e^{A_{c} v_{i}}-I}{v_{i}}
$$

where $v_{i}$ denotes the sampling step and $A_{d_{i}} \rightarrow A_{c}$, when $v_{i} \rightarrow 0$ and matrices $B_{d_{i}}:=\frac{e^{B_{c} v_{i}}-I}{v_{i}}$ and $C_{d_{i}}:=\frac{e^{C_{c} v_{i}}-I}{v_{i}}$. 
For the periodic sampling scheme applied to the hybrid system [i.e. mixed subsystems (2) and (3)], discrete and continuous time is described as follows.

1) In the case of the periodic sampling of second order implemented to subsystem (2) is defined on the sum of time intervals $\cup_{i=0}^{k}(i T ; i T+d)+\cup_{i=0}^{k}(i T+d,(i+1) T)$ and for discrete subsystem (3), sampling instants are taken from set $t_{i} \in\{0, d, T, T+d, \ldots, k T+d\}$; thus, sampling step $v_{i}=d_{i}$ for even samples and $v_{i}=T-d_{i}$ for odd samples.

2) In the case of the periodic sampling of the $L$ th order implemented to subsystem (2) is defined on the sum of time intervals $\cup_{i=0}^{k}\left(i T ; i T+d_{1}\right)+\cup_{i=0}^{k}\left(i T+d_{1}, i T+d_{2}\right)+\ldots+$ $\cup_{i=0}^{k}\left(i T+d_{L-1},(i+1) T\right)$ and for discrete subsystem (3), sampling instants are taken from set $t_{i} \in\left\{0, d_{1}, d_{2}, \ldots, d_{L-1}, T, T+d_{1}, \ldots, k T+d_{L-1}\right\}$; thus, sampling step $v_{1}=d_{1}$, $v_{2}=d_{2}-d_{1}, v_{3}=d_{3}-d_{2}, \ldots, v_{L-1}=d_{L-1}-d_{L-2}, v_{L}=T-d_{L-1}$.

Problem. The aim of this study is to design a controller $K$ by using the D-decomposition method so that the stability of the system with periodic sampling will be ensured.

Subsystems (2) and (3) with state-feedback controller $K$ are controlled by:

$$
\begin{aligned}
& u(t)=K_{c} y(t)=K_{c} C_{c} x(t) \\
& u\left(t_{k}\right)=K_{d} y\left(t_{k}\right)=K_{d} C_{d} x\left(t_{k}\right)
\end{aligned}
$$

The closed-loop system, which consists of subsystems (2) and (3), has the following form:

$$
\begin{aligned}
& \dot{x}(t)=\left(A_{c}+B_{c} K_{c} C_{c}\right) x(t) \\
& y(t)=C_{c} x(t) \\
& x\left(t_{k}+1\right)=\left(A_{d_{i}}+B_{d_{i}} K_{d_{i}} C_{d_{i}}\right) x\left(t_{k}\right) \\
& y\left(t_{k}\right)=C_{d_{i}} x\left(t_{k}\right)
\end{aligned}
$$

where continuous-time subsystem (2) occurs in $t \neq t_{k}$ and a discrete update of the state occurs for $t=t_{k}$ as in (3). The connection between the system matrices of both subsystems is descri-bed by (4).

The D-decomposition set of stabilizing matrices $K$ for the state-space form of the system (6) is described by:

$$
D=\{K \in \mathscr{K}: A+B K C \text { is stable }\}
$$

Thus, set $D$ contains all matrices $K \in \mathscr{K}$ such that $A+B K C$ is stable. Also, matrix $A+B K C$ is stable if all eigenvalues are in the open left-half plane for a continuous-time system and all eigenvalues are in the open unit disc for a discrete-time system; see [6]. Furthermore, assume that matrix $A$ does not have zero or imaginary eigenvalues for the continuous-time subsystem (2) and does not have eigenvalues on the unit circumference for the discrete one (3). 
The D-decomposition technique is based on the decomposition of the parameter space. For systems in the state-space form class $\mathscr{K}$ of parameters $K \in \mathbb{R}^{r \times m}$ matrices, $K$ may be described in many different ways. The simplest cases (see $[6,7])$ are given by:

$$
K=k \text { or } K=k^{T},
$$

where $k \in \mathbb{R}^{n}$, for the case of $m=1$ or $r=1$.

$$
K=k I, k \in \mathbb{R} \text { or } k \in \mathbb{C},
$$

where $I$ - identity matrix, for the case of $m=r$

$$
K \in \mathbb{R}^{2 \times 2}
$$

where matrices $K$ 's dimensions depend on the dimensions of system matrices $B$ and $C$.

Let us consider class (9) where $K=k I$; then, matrix $A+B K C$ is defined as $A+k B C$ due to the fact that $k$ is a scalar value in this case.

Definition $1[6,7]$. For $l=0, \ldots, n$, the $D$-decomposition is the decomposition of the parameter space into regions $D_{l}=\{k \in \mathscr{K}: A+k B C$ has $l$ stable eigenvalues $\}$. The equation describing the boundary of regions $D_{l}$ is called the D-decomposition equation.

Theorem 1 [6,7]. The D-decomposition equation for continuous-time systems is

$$
\operatorname{det}\left(A_{c}+k F_{c}-j \omega I\right)=0, \omega \in(-\infty,+\infty)
$$

where $F_{c}=B_{c} C_{c}$ and for discrete systems with $F_{d_{i}}=B_{d_{i}} C_{d_{i}}$

$$
\operatorname{det}\left(A_{d_{i}}+k F_{d_{i}}-e^{j \omega} I\right)=0, \omega \in[0,2 \pi)
$$

defines the D-decomposition for class $\mathscr{K}$; i.e., if $Q \subset \mathscr{K}$ is a connected set and det $\left(A_{c}+\right.$ $\left.k F_{c}-j \omega I\right) \neq 0, \omega \in(-\infty,+\infty), \forall K \in Q \operatorname{or} \operatorname{det}\left(A_{d_{i}}+k F_{d_{i}}-e^{j \omega} I\right) \neq 0, \omega \in[0,2 \pi), \forall K \in Q$, then $A+B K C$ has the same number of stable and unstable eigenvalues for all matrices $K$ in $Q$.

Proof. The proof is similar to that presented in [9].

The D-decomposition equation allows us to plot a D-curve that assigns regions on a parameter plane where the characteristic equation roots are grouped in a special manner. The boundaries of the regions are received by mapping the $s$-plane in $j w$-axis in the characteristic equation.

System (6) can be also defined as a transfer function in following manner (see [7]):

$$
G(s)=C_{c}\left(A_{c}-s I\right)^{-1} B_{c}
$$

for the continuous-time case; and for the discrete-one:

$$
G(z)=C_{d_{i}}\left(A_{d_{i}}-z I\right)^{-1} B_{d_{i}}
$$

and $\left(A_{d_{i}}-z I\right) \rightarrow\left(A_{c}-s I\right)$ when $v_{i} \rightarrow 0$. 
For the case of the class given by (9), the D-decomposition equation for the transfer functions obtained in (13) and (14) is reduced to the polynomial case; and for continuous-time, it follows that

$$
a(j \omega)+k b(j \omega)=0
$$

where the transfer function is in the form of $G(s)=\frac{b(s)}{a(s)}$ and $w(s)=a(s)+k b(s)$ is the characteristic polynomial. In the discrete-time case, equation (15) has the following form:

$$
a\left(e^{j \omega}\right)+k b\left(e^{j \omega}\right)=0
$$

For further information, see [6] (for example).

\section{D-decomposition for systems with implemented periodic sampling}

In this section, the results of the D-decomposition obtained during the simulations are presented. Simulations were done for system (6) with a periodic sampling of the $L$ th order.

Example 1. Let us take into consideration a closed-loop linear system with an implemented periodic sampling of the $L$ th order with a DC (Direct Current) motor as a plant. The DC motor parameters were taken from [10] as in Table 1. The considered DC motor (along with the indicated parameters) is presented in Figure 3.

Table 1

Parameters of DC motor

\begin{tabular}{|l|l|}
\hline \multicolumn{1}{|c|}{ Parameter } & \multicolumn{1}{c|}{ Value } \\
\hline Armature Resistance & $R_{a}=11.200 \Omega$ \\
\hline Armature Inductance & $R_{a}=0.122 \mathrm{H}$ \\
\hline Rotor Inertia & $J_{m}=0.022 \mathrm{~kg} \cdot \mathrm{m}^{2}$ \\
\hline Viscour Friction Coefficient & $B_{m}=0.003 \frac{\mathrm{N} \cdot \mathrm{m}}{\mathrm{s} \cdot \mathrm{rad}}$ \\
\hline Motor Torque Constant & $k_{m}=1.280 \frac{\mathrm{N} \cdot \mathrm{m}}{A}$ \\
\hline Back Emf Constant & $k_{b}=1.280 \frac{\mathrm{V} \cdot \mathrm{s}}{\mathrm{rad}}$ \\
\hline
\end{tabular}

The state-space form for the continuous-time subsystem is described as (2), and matrices $A_{c}, B_{c}, C_{c}, D_{c}$ are defined on set $\cup_{\beta=0}^{k}(i \cdot 0.10 ; i \cdot 0.10+0.01)$ $+\cup_{\beta=0}^{k}(i \cdot 0.10+0.01, i \cdot 0.10+0.03)+\cup_{\beta=0}^{k}(i \cdot 0.10+0.03 ; i \cdot 0.10+0.06$ $+\cup_{\beta=0}^{k}(i \cdot 0.10+0.06 ;(i+1) \cdot 0.10+\ldots$ by

$$
A_{c}=\left[\begin{array}{cc}
-91.95 & -622.95 \\
1.00 & 0.00
\end{array}\right], B_{c}=\left[\begin{array}{l}
1.00 \\
0.00
\end{array}\right], C_{c}=\left[\begin{array}{c}
0.00 \\
476.90
\end{array}\right], D_{c}=0
$$




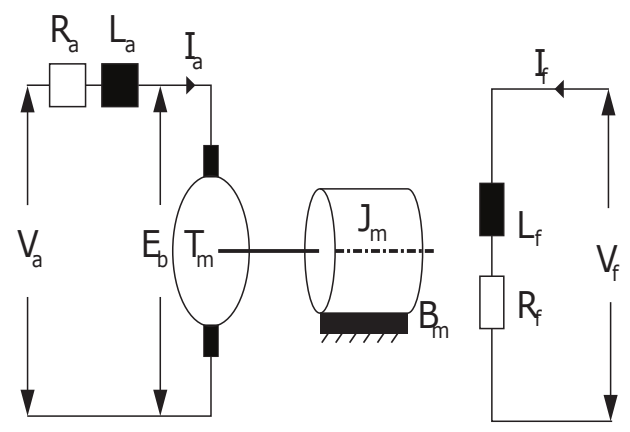

Fig. 3. Exemplary DC motor

Periodic sampling of the 4th order was implemented into the DC motor system with sampling parameters such that $d_{1}=0.01 \mathrm{~s}, d_{2}=0.03 \mathrm{~s}, d_{3}=0.06 \mathrm{~s}$ and $T=0.10 \mathrm{~s}$. The general state-space form of this discrete subsystem is given by (3) and according to the (4) discrete-time system matrix changes in each sampling step. The sampling pattern that is used generates four different sampling steps: $v_{1}=0.01, v_{2}=0.02, v_{3}=0.03$, and $v_{4}=0.04$. These consecutive sampling steps repeat periodically. Thus, four discrete matrices were obtained:

$$
\begin{aligned}
& A_{d_{1}}=\left[\begin{array}{cc}
-100.00 & 0.00 \\
2.72 & -99.00
\end{array}\right], A_{d_{2}}=\left[\begin{array}{cc}
-50.00 & 0.00 \\
2.72 & -49.00
\end{array}\right], A_{d_{3}}=\left[\begin{array}{cc}
-33.33 & 0.00 \\
2.72 & -32.33
\end{array}\right], \\
& A_{d_{4}}=\left[\begin{array}{cc}
-25.00 & 0.00 \\
2.72 & -24.00
\end{array}\right]
\end{aligned}
$$

and the sampling time instants follows $t_{k} \in\{0 ; 0.01 ; 0.03 ; 0.06 ; 0.10 ; \ldots ; i \cdot 0.10\}$.

The D-decomposition for system (6) with $L$ th-order periodic sampling is obtained from the D-decomposition equations given by (13) and (14).

The parametric curve for continuous-time subsystem with matrices $A_{c}, B_{c}, C_{c}, D_{c}$ is given by $k(\omega)=\frac{\omega^{2}-91.95 j \omega-622.95}{476.90}$.

The parametric curves for the discrete-time subsystems are as follows:

$$
\begin{aligned}
& k_{1}\left(e^{j \omega}\right)=\frac{-99 e^{2 j \omega}-9900 e^{j \omega}}{1297.17}, \\
& k_{2}\left(e^{j \omega}\right)=\frac{-49 e^{2 j \omega}-2450 e^{j \omega}}{1297.17}, \\
& k_{3}\left(e^{j \omega}\right)=\frac{-32.33 e^{2 j \omega}-1077.56 e^{j \omega}}{1297.17}, \\
& k_{4}\left(e^{j \omega}\right)=\frac{-24 e^{2 j \omega}-600 e^{j \omega}}{1297.17} .
\end{aligned}
$$

The D-decomposition curve for the continuous-time subsystem is depicted in Figure 4 and for the discrete-time subsystem in Figure 5. 


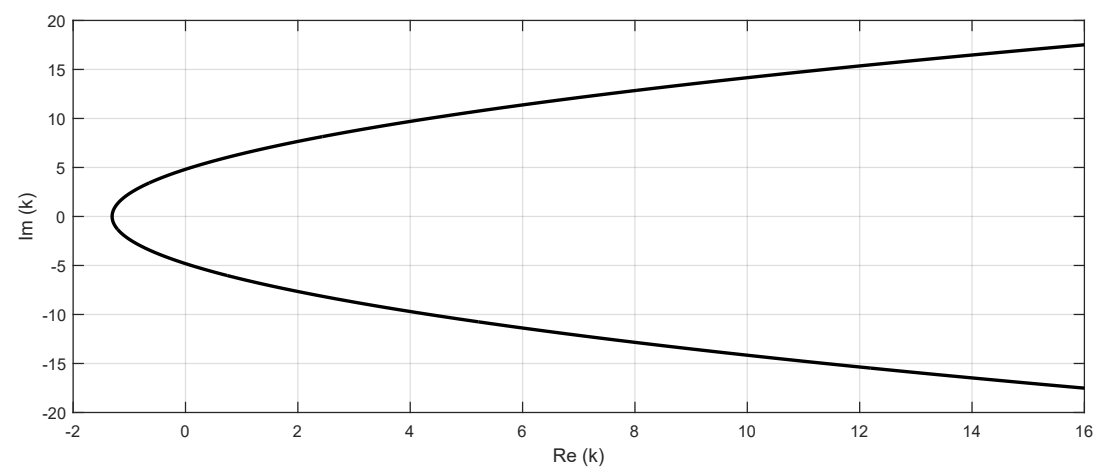

Fig. 4. D-decomposition regions for continuous-time subsystem

In Figure 5, it can be seen that the D-decomposition circle stability regions become smaller for larger sampling steps; for $v_{4}=0.04$, the stability region has the smallest surface.

a)

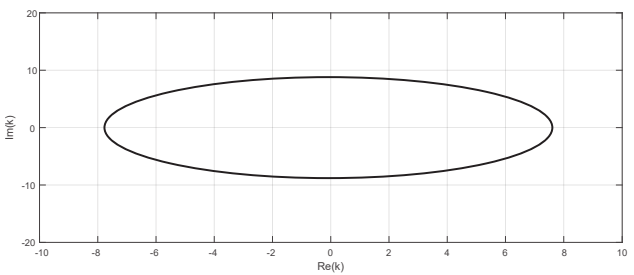

c)

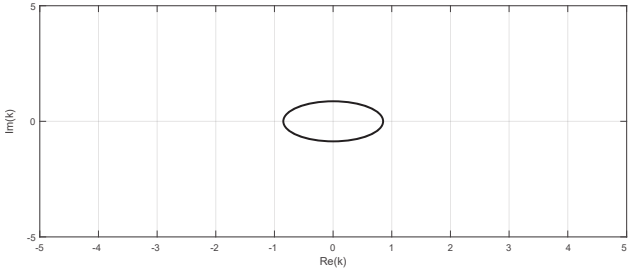

b)

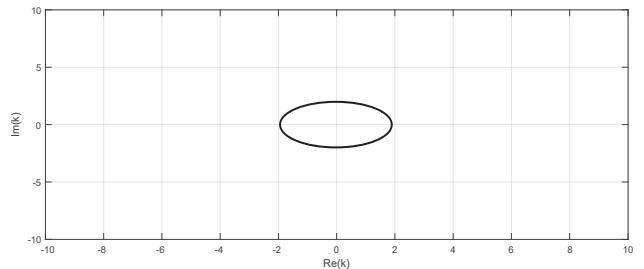

d)

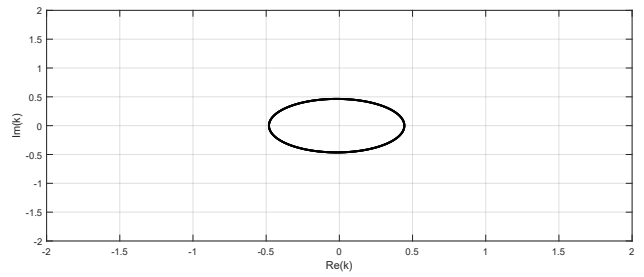

Fig. 5. D-decomposition regions for discrete-time subsystem: a) discrete-time for $v_{1}$; b) discrete-time for $v_{2} ;$ c) discrete-time for $v_{3}$; d) discrete-time for $v_{4}$

It also can be seen that the discrete stability regions are inside the stability region for the continuous-time subsystem. Thus, to achieve stability in system (6), parameter $k$ should be chosen from the smallest circle. Thus, the designed controller is $k=0.2$ (for example). Now, it is necessary to check whether $A+k B C$ has stable eigenvalues for the chosen $k$ value.

For the continuous-time subsystem and matrices $A_{c}, B_{c}, C_{c}, D_{c}$, the eigenvalues are

$$
\lambda_{1}=-85.80, \lambda_{2}=-6.15
$$

Thus, system (6) is stable for the chosen $k=0.2$. 


\section{Conclusions}

In the paper, a sampled-data control system with periodic sampling of the 4th order was implemented as two subsystems - one continuous and one discrete.

The technique of D-decomposition was applied into two subsystems. In each subsystem, one stability region of parameter $k$ was obtained. The stability regions were acquired by taking common parts of their subsystems. It is observed that the D-decomposition curve for discrete subsystems with a smaller sampling step has a wider range than for a discrete subsystem with a greater sampling step.

Future work will include the application of D-decomposition into a continuous-time system with a discrete, non-uniformly sampled controller.

\section{References}

[1] Ben-Romdhane P., Rebai C., Ghazel A., et al., Non-uniform sampling schemes for IF sampling radio receiver, International Conference in Tunisia, 2016, pp. 15-20.

[2] Lin Y., Vaidyanathan P.P., Periodically nonuniform sampling of bandpass signals, IEEE Transactions on Circuits and Systems II: Analog and Digital Signal Processing, 45(3), 1998, pp. 340-351.

[3] Guo G., Systems with nonequidistant sampling: controllable? observable? stable?, Asian Journal of Control 7, 2005, pp. 455-461.

[4] Feizi S., Angelopoulos G., Goyal V., Mdard M., Energy-efficient time-stampless adaptive nonuniform sampling, in: Proceedings of the IEEE Sensors, Limerick, 2011, pp. 912-915.

[5] Eng F., Non-uniform sampling in statistical signal processing, PhD Thesis, 2007, pp. 7-29.

[6] Gryazina E.N., Polyak B.T., Tremba A.A., D-decomposition technique state-of-the-art, Automation and Remote Control 69, 2008, pp. 1991-2026.

[7] Gryazina E.N., The D-decomposition theory, Automation and Remote Control 65, 2004, pp. 1872-1884.

[8] Lennartson B., Middleton R., Christiansson A.-K., Unified, periodic and sampled data $H_{\infty}$ control using delta operator, in: Proceedings of 43rd Conference on Decision and Control, Atlantis, Bahamas, 2004, pp. 2364-2369.

[9] Gryazina E.N., Polyak B.T., Stability regions in the parameter space: D-decomposition revisited, Automatica 42(1), 2006, pp. 13-26.

[10] Suman S.K., Giri V.K., Speed control of DC motor using optimization techniques based on PID controller, IEEE International Conference on Engineering and Technology, 2016, pp. 581-587.

\section{D-podział systemów próbkowanych periodycznie}

Streszczenie: W artykule przedstawiono rozważania na temat stabilności systemów próbkowanych niejednorodnie. W tym celu wykorzystano metodę D-podziału do określenia regionów stabilności w przestrzeni parametrycznej. Ponadto przytoczono podstawowe informacje dotyczące próbkowania niejednorodnego, w szczególności próbkowania periodycznego. Bazując na otrzymanych wynikach symulacji, dokonano porównania systemów z różnymi schematami próbkowania.

Słowa kluczowe: próbkowanie periodyczne, systemy hybrydowe, metoda D-podziału badania stabilności 